\title{
Effect of intense chirped pulses on the coherent phonon generation in Te
}

\author{
O. V. Misochko a) \\ Institute of Solid State Physics, Russian Academy of Sciences, 142432 Chernogolovka, Moscow region, \\ Russia \\ T. Dekorsy \\ Physics Department, Konstanz University, 78457 Konstanz, Germany \\ S. V. Andreev, V. O. Kompanets, Yu. A. Matveets, A. G. Stepanov, and S. V. Chekalin \\ Institute of Spectroscopy, Russian Academy of Sciences, 142190 Troitsk, Moscow region, Russia
}

\begin{abstract}
The authors have studied the influence of chirped laser pulses on the coherent phonon generation in single crystal Te. They have shown that the pulse chirp affects the amplitude of coherent phonons with $A_{1}$ symmetry in the case of intense excitation only. By varying the chirp of an intense exciting pulse, the authors demonstrated that negatively chirped pulses are almost twice more effective in the creation of lattice coherence than positively chirped pulses.
\end{abstract}

The advances in ultrafast laser technology enabled the generation of optical pulses with durations shorter than the period of a lattice vibration in solid state. Hence the use of such ultrashort pulses made it possible to study structural changes in solids that occur on the femtosecond time scale. Coherently generated lattice dynamics in solid state referred to as coherent phonons has been observed in various solids. ${ }^{1-5}$ In transparent materials, the generation mechanism was identified as impulsive stimulated Raman scattering. ${ }^{2}$ In opaque materials, where the linear absorption is significant, the mechanism was initially ascribed to displacive excitation of coherent phonons; ${ }^{1,6}$ however, later it was suggested that this mechanism could be also described as a resonant case of a Raman-like process. ${ }^{3}$ Since ultrashort pulses required for the creation of lattice coherence intrinsically consist of a broad spectrum, the introduction of positive or negative linear chirp on these pulses can systematically change the relative phase of the frequency component. For the time being, nothing is known on how chirped pulses affect the generation of lattice coherence in opaque solids.

In this letter, we study the effects of linear chirp of an intense femtosecond pulse on the coherent phonon generation in the narrow band-gap semiconductor Te which was one of the materials underlying the introduction of the displacive excitation mechanism. ${ }^{1,6}$ The observed dependence of the coherent amplitude of $A_{1}$ symmetry phonons on the pulse chirp demonstrates a significant effect on coherent vibrational amplitude at high intensity excitation which is absent at low intensity excitation.

The sample is a single crystal of Te. Its crystal structure $D_{3}^{4}$ consists of three-atom per turn helices whose axes are arranged on a hexagonal lattice. The fully symmetric $A_{1}$ phonon $(3.6 \mathrm{THz})$ is a "breathing" mode of the lattice for which the helical radius changes leaving the interhelical distance and $c$-axis spacing intact, thereby preserving the lattice symmetry. We coherently excited $A_{1}$ phonons with a pump polarized along the trigonal axis. ${ }^{7}$

Excitation and detection of coherent phonons were carried out with a conventional pump-probe setup. A mode-

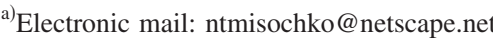

locked Ti:sapphire laser at $800 \mathrm{~nm}$ was amplified using a regenerative $1 \mathrm{kHz}$ amplifier. The final laser pulse had a maximum pulse energy of $\approx 0.7 \mathrm{~mJ}$ and duration of $40 \mathrm{fs}$ at the sample surface. The typical peak power was $0.4 F_{\text {th }}$, where $F_{\text {th }}$ is the threshold above which we observed visible damage to the sample. The pump power exceeded that of the probe by approximately 20 times. The amount and sign of the pulse chirp were changed by detuning the compressor in the amplifier relative to the position corresponding to the minimum duration of transform limited pulses. Both the pump and probe pulses were equally chirped. The laser chirp $\alpha$ was evaluated through the change of pulse duration measured by an autocorrelator, $\alpha= \pm 0.36 \tau_{0} \sqrt{\tau_{c}^{2}-\tau_{0}^{2}}$, where $\tau_{0}$ is the duration of the transform limited pulse and $\tau_{c}$ the duration of the chirped pulse. Both the pump and probe beams were kept close to normal incidence, polarized perpendicular to each other, and focused to spot diameters of 100 and $40 \mu \mathrm{m}$, respectively. A stepper motor controlled the time delay and for the detection we modulated the pump beam at $0.5 \mathrm{kHz}$ with a mechanical chopper.

In an isotropic detection, where diagonal elements of the Raman tensor are sampled, the transient reflectivity of $\mathrm{Te}$ consists of two contributions, see Fig. 1. The oscillations due to coherent phonons are superimposed on an exponential background arising from electronic excitations. For clarity, this electronic contribution is removed in the following from the total signal to isolate the phonon oscillations shown in the upper inset of Fig. 1. To separate the oscillatory component, $\left(\Delta R / R_{0}\right)_{\text {osc }}$, from the electronic relaxation, $\left(\Delta R / R_{0}\right)_{\text {rel }}$, we fit the total signal $\Delta R / R_{0}$ to

$$
\begin{aligned}
\frac{\Delta R}{R_{0}}= & H(t)\left[\left(\frac{\Delta R}{R_{0}}\right)_{\text {rel }} \exp \left(\frac{-t}{\tau_{\text {rel }}}\right)\right. \\
& \left.+\left(\frac{\Delta R}{R_{0}}\right)_{\text {osc }} \exp \left(\frac{-t}{\tau_{\text {osc }}}\right) \sin (\Omega t+\varphi)\right],
\end{aligned}
$$

where $H(t)$ is the Heaviside step function convolved with the pump pulse. The strong modulation of the reflectivity through coherent $A_{1}$ oscillations with a frequency of $3.55 \mathrm{THz}$ (see Fourier-transformed spectrum, lower inset of Fig. 1) is characteristic of the material. ${ }^{1,6-9}$ For a weaker 


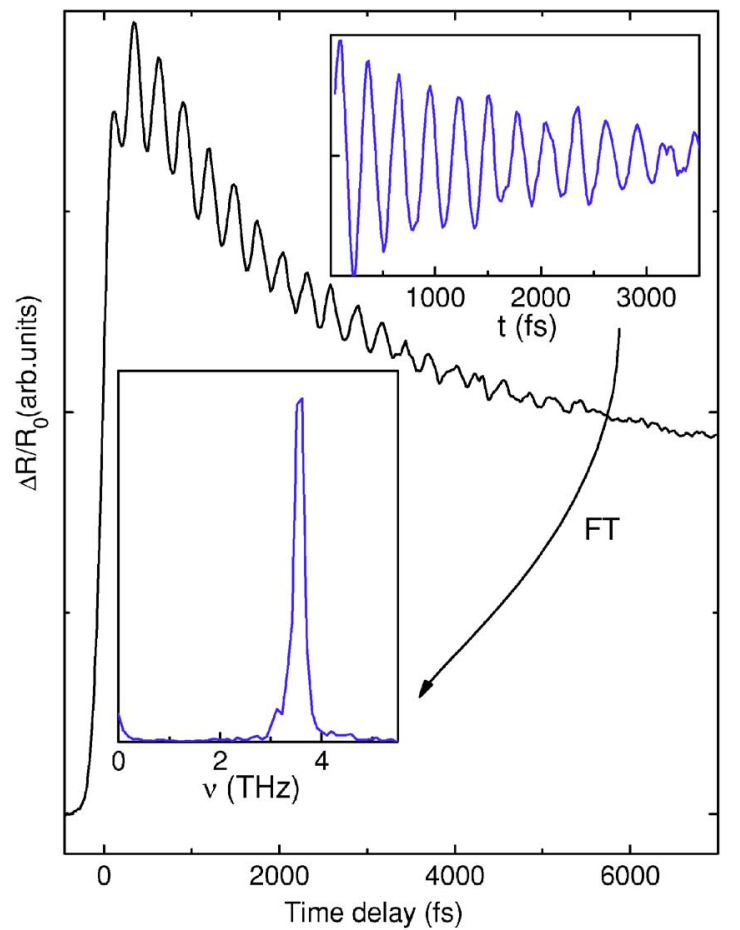

FIG. 1. (Color online) Reflectivity change in Te vs time delay. The upper inset shows the oscillatory part and the lower inset its power Fourier transform.

excitation, the oscillatory frequency is $3.6 \mathrm{THz}$, in agreement with spontaneous Raman experiments. ${ }^{10}$ The bit lower frequency observed in our experiment is due to electronic softening ${ }^{11}$ of the $A_{1}$ mode induced by a high excited carrier density. 8,9

In order to study coherent oscillation properties as a function of chirp, we measured the transient reflectivity signal with differently chirped pulses, as shown in Fig. 2(a) for an unchirped pulse and two large chirp values. The overall rise time of the signal depends on the chirp due to the longer pulse duration associated with longer pulses, see Fig. 2(b). While this rise time slightly depends on the sign of the chirp, being larger for positive chirp, the electronic contribution remains essentially unchanged for longer time delays. For each chirp value, the experimental oscillatory curve was fitted with the damped harmonic function to extract the coherent amplitude, $\left(\Delta R / R_{0}\right)_{\mathrm{osc}}$, oscillation frequency $\nu$, and dephasing time $\tau_{\text {osc }}$. In Fig. 3 we show an example of the fit for two different chirp values. Upon increasing the absolute value of the chirp, the oscillation frequency remains unchanged at the value observed with transform limited pulses. For positively chirped pulses a small $(\leqslant 15 \%)$ but measurable increase in the dephasing time is observed. The amplitude of the oscillations exhibits the strongest dependence on the chirp. Figure 4 shows that the $A_{1}$ oscillations disappear for larger chirp values in a way depending on the sign of the chirp. An intense pump pulse with negative chirp leads to a considerably larger coherent $A_{1}$ amplitude than observed for positively chirped excitation. The overall decrease in coherent amplitude is due to the longer duration of the chirped pulses. However, the observation that, for a given pulse duration, positively or negatively chirped pulses yield very different coherent amplitudes (and somewhat different dephasing times) indicates that these effects are not just due to the temporal broadening of the pulses.
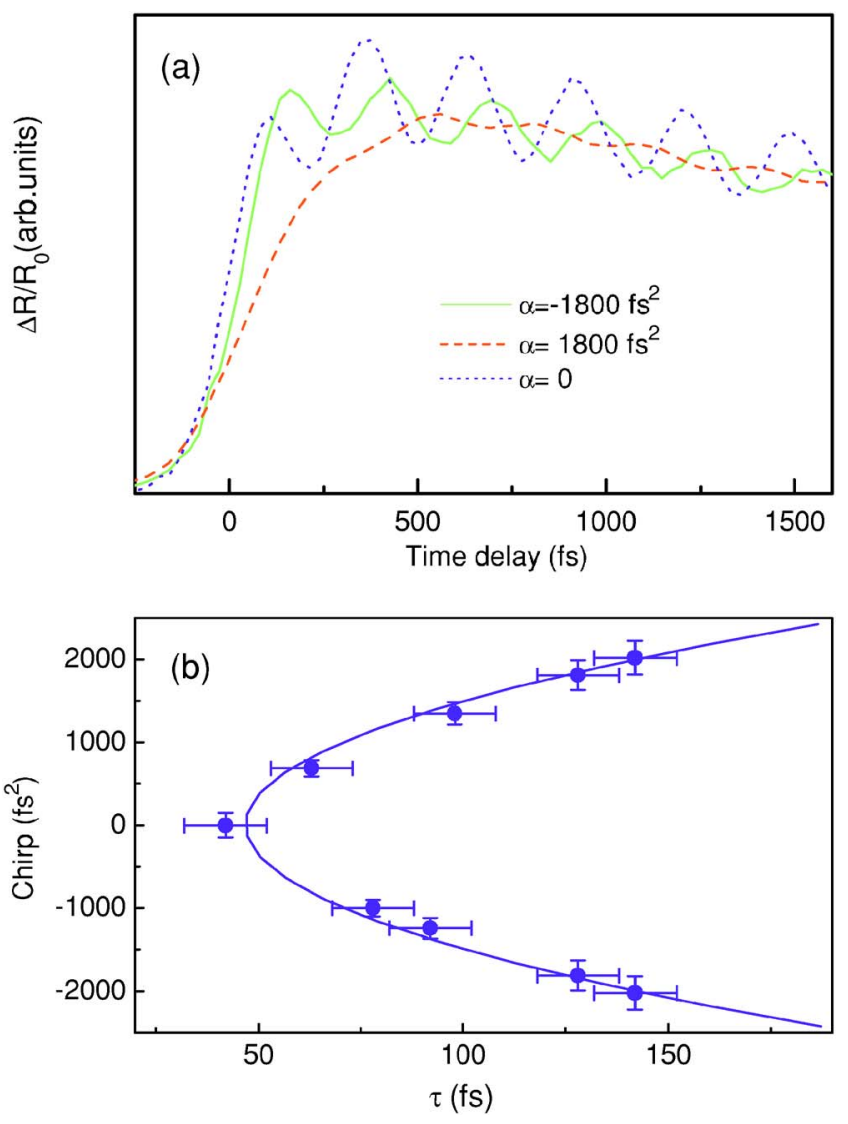

FIG. 2. (Color online) (a) Reflectivity change in Te vs time delay for transform limited, as well as for negatively and positively chirped pulses. (b) Pulse chirp vs duration of the pulse.

Based on the analogy with molecular systems ${ }^{12-15}$ an explanation of the effect of chirped pulses in exciting lattice coherence may be as follows. The full structure of the laser pulse can be viewed in a two-dimensional representation, where one axis is optical frequency and the other is time. A positively chirped pulse has a positive slope in such a plot, with frequency increasing as a function of time, i.e., the low frequency components arrive first, while a negatively chirped pulse has the opposite ordering. Generally, ultrashort optical pulses create nonstationary vibrational states on both the ground and excited electronic states. The nonstationary state created on the ground electronic state is the result of a Raman-like process that induces a small, directed momentum kick on the ground state. On the other hand, the nonstationary state created on the excited electronic state leads to a sudden change in the free energy of the lattice, which responds to the new electronic state by moving toward a new equilibrium position. ${ }^{6}$ A negatively chirped pulse, where low frequencies trail high, leaves less population on the excited state due to a "pump-dump" process, whereas a positively chirped pulse discriminates against this process and maximizes the amount of population left on the excited state. In other words, a decrease in spontaneous emission with negative chirp is accompanied by an increase in stimulated emission due to the dump part of the pulse. However, it cannot be excluded that higher-order interactions (two-photon or excited state absorption ${ }^{15}$ ) are essential for any intensitydependent effects such as those observed. Indeed, we observe that the weaker the pump intensity, the smaller the asymmetry in the coherent amplitude's dependence on the 


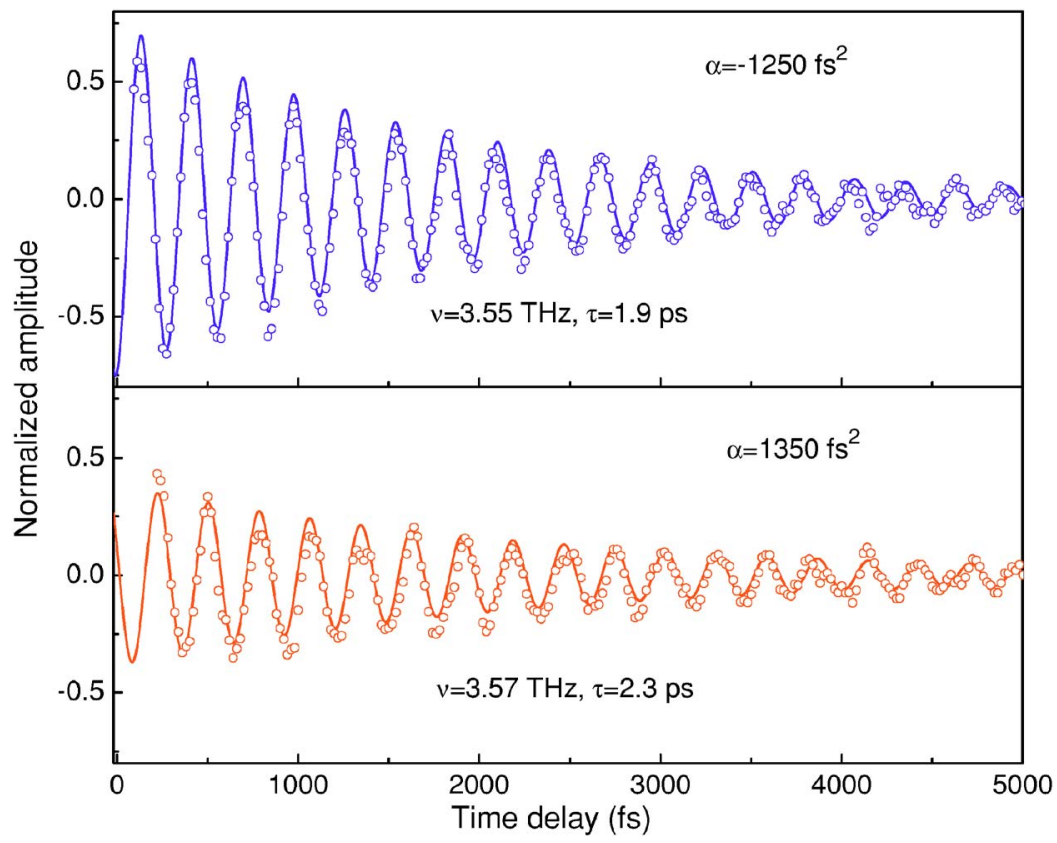

FIG. 3. (Color online) Coherent $A_{1}$ oscillations in Te for negatively and positively chirped pulses. Open symbols are experimental data and solid lines are fits using a damped harmonic function.

chirp sign. At a low excitation level attained with unamplified laser pulses, the asymmetry completely disappears. This observation suggests that the chirp effects are important only at higher intensities where multiphoton interactions become more likely, whereas at low intensities, the phase of the pulse is unimportant.

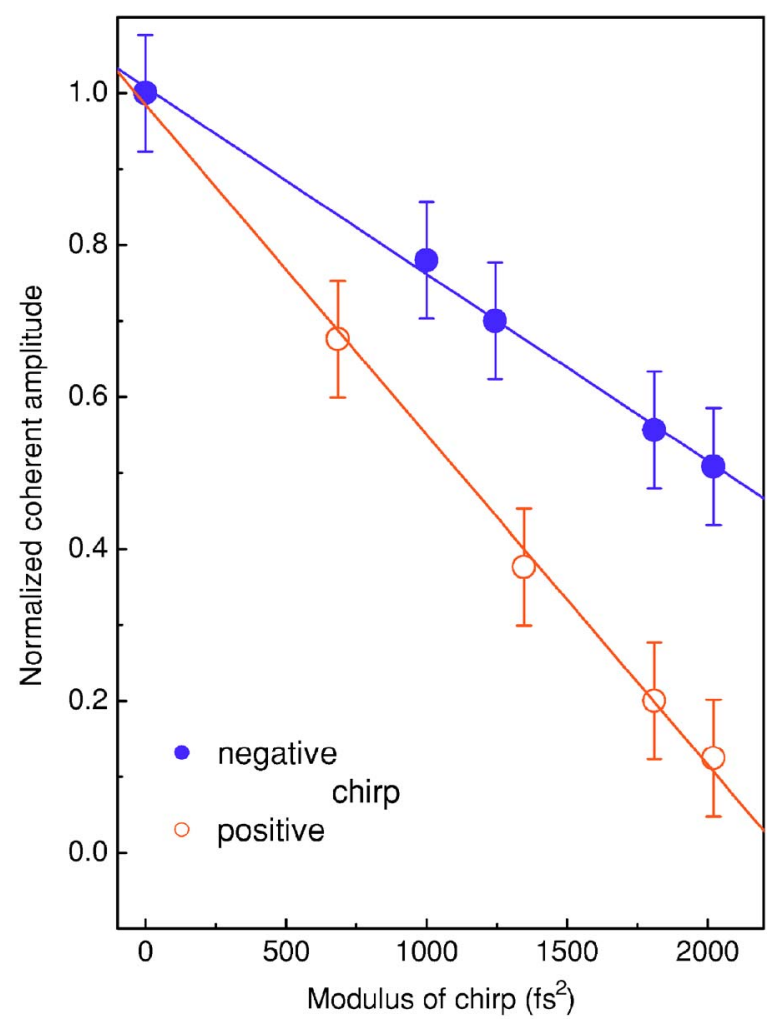

FIG. 4. (Color online) Chirp dependence of the measured coherent $A_{1}$ amplitude. The amplitude is normalized to the excitation conditions with the transform limited pulse.
In conclusion, we have studied the influence of laser chirp on the generation of coherent phonon in the narrow band-gap semiconductor Te. The experiment showed that the intense negatively chirped pulse generates stronger coherent $A_{1}$ oscillations than the positively chirped pulse of the same duration. At the same time, for low intensity excitation the coherent oscillations are independent of chirp.

The authors thank P. Grosse and M. Wuttig for providing them with single crystal Te. This work was supported by the Deutsche Forschungsgemeinschaft (Grant Nos. DE567/9 and 436RUS) and by the Russian Foundation for Basic Research (06-02-16186-a).

${ }^{1}$ T. K. Cheng, J. Vidal, H. J. Zeiger, G. Dresselhaus, M. S. Dresselhaus, and E. P. Ippen, Appl. Phys. Lett. 59, 1923 (1991).

${ }^{2}$ L. Dhar, J. A. Rogers, and K. A. Nelson, Chem. Rev. (Washington, D.C.) 94, 167 (1994).

${ }^{3}$ R. Merlin, Solid State Commun. 102, 207 (1997).

${ }^{4}$ T. Dekorsy, G. C. Cho, and H. Kurz, in Light Scattering in Solids, edited by M. Cardona and G. Güntherodt (Springer, Berlin, 2000), Vol. VIII, p. 169.

${ }^{5}$ O. V. Misochko, Zh. Eksp. Teor. Fiz. 119, 285 (2001) [JETP 92, 246 (2001)].

${ }^{6}$ H. J. Zeiger, J. Vidal, T. K. Cheng, E. P. Ippen, G. Dresselhaus, and M. S. Dresselhaus, Phys. Rev. B 45, 768 (1992).

${ }^{7}$ O. V. Misochko, M. V. Lebedev, and T. Dekorsy, J. Phys.: Condens. Matter 17, 3015 (2005).

${ }^{8}$ S. Hunsche, K. Wienecke, T. Dekorsy, and H. Kurz, Phys. Rev. Lett. 75, 1815 (1995).

${ }^{9}$ C. A. D. Roeser, M. Kandyla, A. Mendioroz, and E. Mazur, Phys. Rev. B 70, 212302 (2004).

${ }^{10}$ A. S. Pine and G. Dresselhaus, Phys. Rev. B 4, 356 (1971).

${ }^{11}$ P. Tangney and S. Fahy, Phys. Rev. B 65, 054302 (2002).

${ }^{12}$ B. Kohler, V. V. Yakovlev, J. Chi, J. L. Krause, M. Messina, K. R. Wilson, N. Schwentner, R. M. Whitnell, and Y. Yan, Phys. Rev. Lett. 74, 3360 (1995).

${ }^{13}$ C. J. Bardeen, Q. Wang, and C. V. Shank, Phys. Rev. Lett. 75, 3410 (1995).

${ }^{14}$ E. M. Hiller and J. A. Cina, J. Chem. Phys. 105, 3419 (1996).

${ }^{15}$ D. J. Tannor and S. A. Rice, J. Chem. Phys. 83, 5013 (1985). 